\title{
Consensus on the treatment of autoimmune bullous dermatoses: dermatitis herpetiformis and linear IgA bullous dermatosis - Brazilian Society of Dermatology*
}

\author{
Everton Carlos Siviero do Vale ${ }^{1}$, Oscar Cardoso Dimatos ${ }^{2}$, Adriana Maria Porro ${ }^{3}$, Claudia Giuli Santi ${ }^{4}$
}

DOI: http:/ / dx.doi.org/10.1590/abd1806-4841.2019940208

\begin{abstract}
Dermatitis herpetiformis and linear IgA bullous dermatosis are autoimmune diseases that present with pruritic urticarial papules and plaques, with formation of vesicles and blisters of subepidermal location, mediated by IgA antibodies. Mucosal lesions are present only in linear IgA bullous dermatosis. The elaboration of this consensus consisted of a brief presentation of the different aspects of these dermatoses and, above all, of an updated literature review on the various therapeutic options that were discussed and compared with the authors' experience, aiming at the treatment orientation of these diseases in Brazil. Dermatitis herpetiformis is a cutaneous manifestation of celiac disease, and can be controlled with a gluten-free diet and dapsone. On the other hand, linear IgA bullous dermatosis arises spontaneously or is triggered by drugs, and can be controlled with dapsone, but often requires the association of systemic corticosteroids and eventually immunosuppressants. Keywords: Celiac disease; Consensus; Dermatitis herpetiformis; Linear IgA bullous dermatosis; Skin diseases, vesiculobullous; Therapeutics
\end{abstract}

\footnotetext{
Received 14 September 2018.

Accepted 30 January 2019.

* Work conducted at the Sociedade Brasileira de Dermatologia, Rio de Janeiro (RJ), Brazil.

Financial support: None.

Conflict of interest: None.

Dermatology Service, Hospital das Clínicas, Universidade Federal de Minas Gerais, Belo Horizonte (MG), Brazil.

Dermatology Service, Hospital Universitário Professor Polydoro Ernani de São Thiago, Universidade Federal de Santa Catarina, Florianópolis (SC), Brazil.

Department of Dermatology, Escola Paulista de Medicina, Universidade Federal de São Paulo, São Paulo (SP), Brazil.

Department of Dermatology, Hospital das Clínicas, Faculdade de Medicina, Universidade de São Paulo, São Paulo (SP), Brazil.
} 


\section{DERMATITIS HERPETIFORMIS}

\section{INTRODUCTION}

Dermatitis herpetiformis (DH), also known as Duhring-Brocq disease, is a chronic autoimmune dermatosis that is caused by a hypersensitivity to gluten, usually manifesting as papulovesicular and eroded/crusted lesions, with intense pruritus and burning, preferentially located on the elbows, knees, and buttocks. Presenting as a cutaneous manifestation of celiac disease (CD), general and gastrointestinal symptoms are rare, although most patients show small bowel villous atrophy. ${ }^{1}$ A definitive diagnosis is made, based on the presence of granular IgA deposits on dermal papillae by direct immunofluorescence, the target antigen of which is the epidermal transglutaminase enzyme. The dermatological condition responds slowly to a gluten-free diet but undergoes prompt resolution with oral dapsone.

\section{EPIDEMIOLOGY}

There are no epidemiological studies on DH in Brazil, but it is considered an uncommon disease in this country, based on the experience of specialists. The prevalence is higher in Northern Europe, especially in Scandinavia, and in individuals of Northern European ancestry in the United States of America but is low among Asians and Africans. Recent studies have shown a prevalence of 30/100,000 in the United Kingdom and 75/100,000 in Finland, with a tendency toward a lower incidence of $\mathrm{DH}$, as opposed to a rising incidence of CD. Between $15 \%$ and $25 \%$ of patients with CD have concomitant $\mathrm{DH}$. Men are more often affected than women, at a proportion of 1.5 to 2:1, but more recent studies have shown that this difference tends to decrease. Children are rarely affected by $\mathrm{DH}$, which usually starts between the ages of 40 and 50 years, although it develops earlier in individuals from southern and eastern Europe, perhaps due to their genetic constitution and eating habits. ${ }^{2}$

\section{ETIOPATHOGENESIS}

In the pathogenesis of $\mathrm{DH}$, genetic, immunological, and environmental factors interact. There is a strong predisposition to $\mathrm{DH}$ in individuals with relatives who are affected by the disease. Hypersensitivity to gluten has a strong genetic component, as demonstrated in cases of monozygotic twins and by recurrente 15 times higher in first-degree relatives than in the general population. Both DH and CD have a close association with alleles of the HLA-DQ2 and HLA-DQ8 haplotypes. The immunological basis of DH is linked to gluten intolerance and CD. Tissue transglutaminase (TG2) is the antigenic target of IgA deposits in the intestinal mucosa in $\mathrm{CD}$, whereas epidermal transglutaminase (TG3) is targeted by IgA deposits on the skin in DH. Autoantibodies against TG2 from the gut then react with TG3 to form immunocomplexes, which deposit on the dermal papillae and trigger a local inflammatory process with neutrophil chemotaxis. ${ }^{3}$

\section{CLINICAL FEATURES}

The morphology and distribution of the lesions are very characteristic. They appear as erythematous papules, urticariform plaques, and grouped vesicles, intensely pruritic, that evolve with erosions, excoriations, and crusts by scratching. Therefore, residual hyperchromia and hypochromia are frequently observed. Bullous lesions are less common in DH. In addition to pruritus, patients may complain of burning and tingling sensations, usually preceding the onset of new lesions. They are distributed symmetrically throughout the extensor surfaces of the upper and lower limbs, particularly on the elbows and knees, in the posterior cervical region, scalp, shoulders, sacral region, and buttocks. Eventually, the condition may disseminate, affecting other body areas. ${ }^{4}$ Purpuric lesions are less common, more often located at the extremities, especially in children. Oral lesions are extremely rare in $\mathrm{DH} .{ }^{5}$

From a clinical point of view, DH should be distinguished primarily from other autoimmune bullous dermatoses, such as bullous pemphigoid and linear IgA dermatosis, in addition to pruritic dermatoses, such as scabies, eczema, prurigo, and urticaria. ${ }^{6}$

Due to malabsorption, $\mathrm{DH}$ patients are at increased risk of developing iron deficiency anemia, megaloblastic anemia, osteopenia, and fractures.

In addition to celiac disease, DH may be associated with other autoimmune diseases, most commonly thyroid disease (Hashimoto thyroiditis, hypothyroidism, and hyperthyroidism), type 1 diabetes, and pernicious anemia, as well as alopecia areata, vitiligo, lupus erythematosus, rheumatoid arthritis, Sjögren syndrome, dermatomyositis, sarcoidosis, multiple sclerosis, and Addison disease. $^{7}$

Although there are several reports of a link to lymphomas, primarily the non-Hodgkin type, the relationship between $\mathrm{DH}$ and the development of lymphoma is a controversial subject. ${ }^{8}$ An epidemiological study of $846 \mathrm{DH}$ patients showed no increased mortality or greater risk of developing lymphomas and intestinal malignancies compared with the general population. ${ }^{9}$

\section{LABORATORY DIAGNOSIS}

For the diagnosis, a lesion specimen, preferably an intact vesicle, should be collected for routine anatomopathological examination, and another sample of perilesional healthy skin should be analyzed by direct immunofluorescence (DIF).

Typical histopathological findings are a subepidermal vesicle with neutrophils in its interior, in addition to neutrophils that form microabscesses in the dermal papillae. These findings are not exclusive to DH and may be seen in other bullous dermatoses. ${ }^{10,11}$ It is important to note that the histopathological condition is usually non-specific in $35 \%$ to $40 \%$ of cases, presenting only as a perivascular lymphocytic infiltrate and minimal inflammation in the dermal papilla. ${ }^{12}$

Conversely, DIF with granular IgA deposits at tips of dermal papillae is the gold standard test for $\mathrm{DH}$ diagnosis, which has a sensitivity of $90 \%$ to $95 \% .{ }^{13,14}$

The detection of circulating autoantibodies completes the diagnosis of the disease, but it is unnecessary. Anti-TG3 antibodies are more sensitive and important in DH, but anti-TG2, antiendomysium, and antigliadin antibodies, which are more related to celiac disease, can be detected. Serum levels of these autoantibodies show 
a correlation with intestinal disease activity and help to monitor adherence to a gluten-free diet, with very low titers or negativity in patients with good adherence to this diet. ${ }^{15}$

Intestinal biopsy is not indicated in a patient with confirmed $\mathrm{DH}$, because it is the cutaneous manifestation of celiac disease. It is also unnecessary as a means to check patient adherence to the diet, which can be assessed easily by observation of skin lesions and serological tests. ${ }^{16}$

\section{TREATMENT}

\section{1- Professionals involved}

Depending on the patient's age and the presence of general and gastrointestinal symptoms, in addition to the dermatologist, intervention by an internist, pediatrician, gastroenterologist, nutritionist, and digestive endoscopist may be needed. ${ }^{17}$

\section{2- General patient and family guidelines}

The patient and family members should be informed about the chronic nature of the disease; its relation to celiac disease, even in the absence of gastrointestinal symptoms; possible disease complications; the importance of adherence to a gluten-free diet to control the dermatological and intestinal conditions; the use of medication and its possible adverse effects; and the prediction of long-term clinical and laboratory follow-up. They should also be encouraged to seek support associations for celiac patients to better educate themselves about diet and to receive support to assist in their adherence. ${ }^{17}$

\section{3- Objectives}

The initial goal comprises the control of dermatological symptoms, such as pruritus and burning, as well as the resolution of cutaneous lesions, which can be attained more quickly with medication. However, the improvement of digestive symptoms, when present, can only be achieved with diet, which is also important for long-term control of the dermatological condition. Intestinal involvement may affect the development of children who are affected by the disease, who should be evaluated using growth curves during follow-up. Emphasis should be placed on adherence to diet as a measure of intestinal disease control, even in asymptomatic patients, to prevent late complications of the disease. ${ }^{18}$

\section{4 - Treatment plan}

\section{a) Gluten-free diet}

Both gastrointestinal, if present, and cutaneous manifestations respond to a gluten-free diet - the former after 3-6 months but the latter after 1-2 years. ${ }^{15}$ Therefore, medications must be combined in the first years of treatment, even in patients with good adherence to diet.

Foods that are derived from wheat, rye, barley, and malt should be discarded, and pure oat consumption is allowed; however, most oat products on the market are contaminated with gluten. Patients should be instructed to consult processed food labels closely for the presence of gluten in their composition and avoid eating foods with unknown ingredients. It is important to remind patients that many excipients, and food and pharmaceutical additives may contain gluten. ${ }^{19}$
Because gastrointestinal symptoms are absent or mild in most cases and because the skin condition is controlled rapidly by medication, it becomes difficult to convince patients to adhere to a strict diet, considering its complexity, cost, and social limitations. Hence, patients should be guided by a nutritionist and participate in support groups to acquire greater knowledge about the diet. Supplementation with iron, folate, and vitamins D and B12 may be needed when their deficiencies are confirmed. Even patients who fail to eliminate but only reduce their gluten intake may gain some benefit, however without complete control of the cutaneous condition, depending on the medication. ${ }^{20,21}$

In addition to resolving cutaneous and gastrointestinal symptoms, a gluten-free diet improves malabsorption, allowing dose reduction and possibly discontinuation of medication and helping prevent the development of lymphomas by lowering persistent antigenic stimulation.

\section{b) Dapsone}

Dapsone is the drug of choice in $\mathrm{DH}$, because it relieves pruritus in a few hours and resolves skin lesions in several days, but on halting this medication, the condition recurs in 24 to 48 hours. ${ }^{18}$ However, it has no effect on intestinal disease or in reducing the risk of lymphoma. ${ }^{8,22}$

Dapsone has anti-inflammatory activity, inhibiting neutrophil chemotaxis, the release of leukotrienes and prostaglandins, and reducing tissue damage that is mediated by neutrophils and eosinophils. ${ }^{23}$

It should be started at a dosage of $50 \mathrm{mg} / \mathrm{d}$, with a gradual increase to $200 \mathrm{mg} / \mathrm{d}$, depending on tolerance and the resolution of the dermatological condition. A dosage of $0.5-1 \mathrm{mg} / \mathrm{kg} / \mathrm{d}$ is usually effective in controlling pruritus and preventing the onset of new lesions in most cases. ${ }^{15}$ The lowest dose that is required to maintain cutaneous disease in remission should be determined and then discontinued when the benefits from the diet are obtained, which usually occurs after 1-2 years. For children, a dosage of $1-2 \mathrm{mg} / \mathrm{kg} / \mathrm{d}$ is recommended.

Laboratory tests, including glucose-6-phosphate dehydrogenase (G6PD), complete blood count, reticulocyte count, liver enzymes, bilirubin, creatinine, and urinalysis, should be performed prior to starting medication. They should be repeated every 2 weeks in the first 3 months of treatment and then every 3 months. ${ }^{24}$

The main adverse effects of dapsone are dose-dependent hemolysis and methemoglobinemia; therefore, most patients usually tolerate it as long as the dose is adjusted individually. Greater caution should be placed on G6PD-deficient patients, in whom acute hemolytic anemia is usually more severe, requiring drug withdrawal. Due to its effect of preventing the oxidative stress that dapsone exerts on red blood cells, some groups advocate the use of vitamin E at a dosage of $800 \mathrm{mg} / \mathrm{d}$ to minimize the risk of hemolytic anemia. ${ }^{25}$ Methemoglobinemia is generally insidious but well tolerated at levels below $20 \%$ and should be suspected in the presence of cyanosis, dizziness, dyspnea, lethargy, and headache. ${ }^{26}$ Cimetidine, at a dose of 400mg, 3-4 times a day, may reduce methemoglobinemia without interfering with the effects of dapsone on $\mathrm{DH} .{ }^{27}$ Other less common side effects include leukopenia, agranulocytosis, hypersensitivity 
reactions, peripheral neuropathy, nephrotic syndrome, and hepatic and pulmonary changes. ${ }^{23}$ Agranulocytosis is rarer, which usually appears within the first 3 months of treatment. Hypersensitivity reactions to dapsone are more severe but rare, presenting as fever, rash, and lymphadenopathy, in addition to systemic involvement to varying degrees, and appear in approximately $1 \%$ of patients after 2-6 weeks of treatment.

Dapsone is contraindicated in patients with sulfa allergy, acute porphyrias, severe anemia, and severe cardiopulmonary disease. ${ }^{28}$ Drug interactions are rare, but the concomitant use of trimethoprim or probenecid may raise plasma levels, increasing the hematological toxicity of dapsone. ${ }^{8}$

With regard to the risk of use in pregnancy, it is classified as a category $\mathrm{C}$ drug (FDA) and therefore is not recommended during pregnancy or breastfeeding. However, the experience of its wide use in leprosy during pregnancy and the absence of deleterious effects in the mother and the fetus indicate that dapsone can be used safely in the gestational period. ${ }^{29}$

\section{c) Alternative drugs}

Sulfasalazine is the second-line drug when it is not possible to use dapsone due to its adverse effects and, less frequently, lack of response. Due to the variable absorption of sulfasalazine, its efficacy is less predictable compared with dapsone. ${ }^{30}$ The usual recommended dosage is $1-2 \mathrm{~g} / \mathrm{d}$, but up to $4 \mathrm{~g} / \mathrm{d}$ may be needed. ${ }^{31}$ The most common adverse reactions are digestive, such as nausea, vomiting, and anorexia, which can be avoided with enteric-coated tablets. Hemolytic anemia, hypersensitivity reactions, proteinuria, and crystalluria are less common. Patients should be monitored with complete blood count and urinalysis before treatment, monthly in the first 3 months and every 6 months thereafter. ${ }^{32}$

Sporadic studies, based on case reports, have demonstrated the beneficial effects of other alternative drugs in $\mathrm{DH}$, such as colchicine, cyclosporin, heparin, tetracycline, and nicotinamide. ${ }^{33-36}$

$\mathrm{DH}$ does not respond to systemic corticosteroids, and antihistamines have a limited effect on pruritus. ${ }^{13}$ Potent topical corticosteroids may provide temporary relief of itching but are only allowed in the acute phase of the disease, until benefits of systemic treatment are obtained. ${ }^{30}$

In refractory cases, immunosuppressants, such as metho- trexate, azathioprine, and mycophenolate mofetil could be indicated. ${ }^{37}$ Immunobiologicals, such as rituximab, are also alternative options for resistant cases, although it has been documented that $\mathrm{DH}$ is triggered by infliximab. ${ }^{38,39}$

\section{5 - Follow-up}

Once the disease is controlled, with good tolerance to medication, the patient can be monitored every 6 months with clinical and laboratory tests. After the medication is withdrawn, if possible, evaluations may be performed annually. Adherence to diet and the possible development of malabsorption, dyslipidemia, and other CD-related complications, such as autoimmune diseases and lymphoma, should also be assessed.

\section{EVOLUTION AND PROGNOSIS}

Up to $20 \%$ of patients who adhere to a gluten-free diet for several years may develop immunotolerance and can return to a normal diet. ${ }^{40,41}$ The remaining patients should keep a gluten-free diet to maintain control of their disease.

As in $\mathrm{CD}$, a recent study has shown that the risk of developing non-Hodgkin lymphoma is increased in $\mathrm{DH} .{ }^{42}$ However, adherence to diet for more than 5 years seems to protect $\mathrm{DH}$ patients against lymphomas. ${ }^{43}$ Similarly, another recent study of 476 patients showed that the mortality rate due to lymphoma was significantly reduced after 5 years of a gluten-free diet, in addition to being lower in comparison to the general population. ${ }^{44}$ A previous study of 846 patients with dietary adherence showed no increased risk of malignancy and a slightly lower mortality rate in $\mathrm{DH} .{ }^{9}$ The long-term prognosis of $\mathrm{DH}$ patients is excellent, but it appears to be related to strict adherence to a gluten-free diet. ${ }^{45}$

\section{CONCLUSIONS}

Dermatitis herpetiformis is considered a cutaneous manifestation of celiac disease, and it should be approached as a primarily intestinal disease. Therefore, despite the absence of digestive manifestations in the majority of patients, adherence to a gluten-free diet is the only effective measure in controlling persistent inflammation of the intestinal mucosa. On the other hand, dapsone can halt the cutaneous manifestations rapidly and can thus be used until they are definitively controlled by a gluten-free diet. 
LINEAR IGA BULLOUS DERMATOSIS

\section{INTRODUCTION}

First described in 1901 by Bowen, linear IgA bullous dermatosis (LABD), also called linear IgA disease, is a rare subepidermal autoimmune bullous disease, characterized by the presence of linear and homogeneous IgA deposits and, occasionally, of IgG, IgM, and C3 in the epidermal basement membrane zone (BMZ). ${ }^{46}$

LABD is not related to gluten-sensitive enteropathy, which affects adults and children. In this age group, LABD is also known as chronic bullous disease of childhood. ${ }^{47}$

\section{EPIDEMIOLOGY}

LABD is a rare disease in adults and children. ${ }^{48}$ Yet, it is the most common autoimmune bullous dermatosis in childhood. ${ }^{49}$ Its incidence rates varies in different regions. ${ }^{50}$ The lowest incidence is in Bavaria, Germany (0.22/million/year). ${ }^{51,52}$ Disease occurrence appears to be the greatest in developing countries in Africa (Tunisia and Uganda), attributed to the distribution of ages in the population, because most of the inhabitants in these countries are minors..$^{50}$

The epidemiological data regarding the distribution of cases with regard to gender vary. There are 2 peaks in incidence: infancy, from six months to six years of age, and adulthood, especially from age 60 years. $^{48}$

\section{ETIOPATHOGENESIS}

It is known that subepidermal blisters are caused by IgA1 autoantibodies against antigens of various molecular weights in the basement membrane zone of the skin and mucosa that is lined with stratified squamous epithelium. ${ }^{52}$

As in many other autoimmune diseases, the underlying etiology and pathophysiological mechanism that triggers the autoimmune response remain largely unknown. ${ }^{48}$

The multitude and complexity of antigens explain the heterogeneity of this disorder in terms of the ultrastructural location of target antigens and epitopes or antigenic source..$^{48}$

The major target antigens are the 120-kDa (LAD-1) and 97kDa (LABD-97) ectodomains of BP180 (collagen XVII), and there are cases with autoantibodies against collagen VII, BP230, a6 $\beta 4$ integrin, laminin, and other proteins. ${ }^{46,53-55}$ Circulating IgA antibodies are present in one third to one half of patients. ${ }^{56}$

The disease pathophysiology involves several inflammatory pathways, such as the activation of the alternative complement pathway; activation of CD4+ lymphocytes, HLA-DR, and CD30+; cytokine synthesis by keratinocytes, such as IL8 and GM-CSF; and recruitment of polymorphonuclear neutrophils and eosinophils. IgA fixation in these polymorphonuclear cells, through Fc receptor - not direct binding to its antigen - induces the release of proteolytic enzymes in situ, such as collagenase and elastase, thus causing dermoepidermal delamination (or detachment). ${ }^{57,58}$

The disease onset may be spontaneous or drug-induced, with vancomycin being the most frequently described trigger. There are also reports of other drugs that cause LABD, such as amiodarone, non-hormonal anti-inflammatories, acetaminophen, captopril, and antibiotics other than vancomycin, such as ceftriaxone, penicillin, and metronidazole. ${ }^{46,48,59}$ It is believed that cross-reaction of drugs with hemidesmosome antigens results from greater exposure of these antigens, alteration of their structure, or the formation of haptens. These events promote IgA production against BMZ antigens, triggering a response that is similar to those in cases of idiopathic LABD. ${ }^{48}$ There are descriptions of LABD cases that have been induced by sun exposure and gestation. ${ }^{55,60}$

Inflammatory diseases and neoplasias have been associated with LABD. Some cases that are related to inflammatory bowel disease, infection, and systemic lupus erythematosus have been described. ${ }^{46}$ However, the significance of these correlations remains unknown. ${ }^{61}$ With regard to neoplasms, lymphoproliferative diseases (non-Hodgkin lymphoma, chronic lymphocytic leukemia) are more frequent, although there are reports of associated solid neoplasms (eg, bladder, thyroid, and esophagus). ${ }^{46,48}$

\section{CLINICAL CONDITION}

Cutaneous manifestations are heterogeneous and can mimic other bullous diseases. There may be erythematous papules, urticarial plaques, or vesiculobullous lesions, ${ }^{46}$ that may be accompanied by pruritus of variable intensity. The latter are generally tense and may have an annular or arcuate configuration or rosette pattern or bead-like appearance when new bullous lesions arise at the periphery of previous lesions. ${ }^{46,62,63}$ This clinical characteristic occurs more frequently in children than in adults. ${ }^{64}$

The disease can affect the flexor surfaces of limbs and chest, as in bullous pemphigoid, or the extensor regions of the limbs, as in dermatitis herpetiformis. ${ }^{46,62}$ Urticated papules, similar to urticaria, and excoriated papulonodular lesions may also be seen, such as in prurigo. ${ }^{63,65}$

In children, the lesions are located primarily on the lower abdomen and perineal area, with significant involvement of the anogenital area. The face, hands, and feet may also be affected. In adults, the lesions arise mainly on the extensor surfaces, chest, gluteal region, and face (commonly, the perioral area)..$^{48}$

Mucosal involvement occurs in most cases (60-80\%). ${ }^{46}$ Any mucous membrane may be affected-most frequently the oral and ocular mucosa. Oral lesions consist of erosions and painful ulcerations, desquamative gingivitis, and cicatricial lesions. ${ }^{48}$ Chronic conjunctivitis can lead to symblepharon and even blindness. ${ }^{66}$

Drug-induced LABD usually has a more severe, extensive, and atypical presentation than spontaneous disease. ${ }^{59}$ There are cases of vancomycin-induced LABD that mimics toxic epidermal necrolysis. $^{67}$

The main differential diagnoses in LABD are dermatitis herpetiformis, bullous pemphigoid, mucous membrane pemphigoid, inherited and epidermolysis bullosa acquisita, bullous lupus erythematosus, bullous impetigo, and erythema multiforme. ${ }^{48,64}$ 


\section{LABORATORY DIAGNOSIS}

The pathological examination reveals the presence of subepidermal blisters with a predominantly neutrophilic infiltrate and eventually eosinophils and mononuclear cells. Microabscesses in the dermal papillae may develop. ${ }^{46}$ In view of these histopathological findings, common to other bullous dermatoses, DIF must be performed, which yields linear and homogeneous deposits of IgA in the BMZ. Occasionally, IgG, IgM, and C3 are found. ${ }^{68-70}$

Indirect immunofluorescence, used to detect circulating antibodies, may be IgA-positive in half of all cases and can be used to rule out other dermatoses. ${ }^{56,69}$ The salt-split technique, in general, shows fluorescence on the epidermal side of the blister. ${ }^{69}$ Dermoepidermal or dermal fluorescence may occur, however, depending on the antigenic target. ${ }^{71}$

\section{TREATMENT} is essential. ${ }^{72}$

In drug-induced cases, suspension of the suspected drug

Topical corticosteroid therapy on the skin or mucous membrane may be very effective for LABD as a single agent in mild disease or as an adjuvant for limiting the systemic therapeutic dosage in more severe disease. ${ }^{48}$

The best options for systemic therapy are sulfones (dapsone) and sulfonamides (sulfapyridine or sulfamethoxypyridazine). As with dermatitis herpetiformis, dapsone is considered the firstline therapy for LABD. Dapsone therapy is best initiated at low dosages ( $25 \mathrm{mg} /$ day in adults) and then gradually increased according to control of the clinical condition, as determined by the emergence of new lesions. ${ }^{57}$ The dosage is $0.5-3 \mathrm{mg} / \mathrm{kg} /$ day for children and $25-150 \mathrm{mg} /$ day for adults. ${ }^{48}$ The side effects of this medicine include hemolytic anemia (especially in patients with G6PD deficiency), leukopenia, methemoglobinemia, and abnormalities of liver function tests. ${ }^{48,57}$ For this reason, it is recommended that G6PD levels be measured before starting therapy with dapsone. If the patient does not present with enzyme deficiency, initial monitoring with blood counts and liver function tests is performed weekly in the first month of treatment and then monthly in the subsequent 6 months. It is also suggested that long-term therapy be monitored every 6 months. ${ }^{48,57}$ Mucosal involvement is typically more resistant to treatment with dapsone alone. ${ }^{72}$

Sulfonamides, including sulfapyridine and sulfamethoxypyridazine, are alternatives to dapsone use and may be used alone or in combination with dapsone. Both drugs have similar profiles compared with the adverse effects of dapsone. As with dapsone use, the same precautions apply to sulfonamides. ${ }^{48,72}$

In some patients, LABD is not adequately controlled by these medications or dapsone alone, but the combination with corticosteroids and immunosuppressants, or both, such as azathioprine, mycophenolate mofetil, cyclosporine, cyclophosphamide, and topical tacrolimus may be necessary, mainly due to the side effects of dapsone, which limits its daily dose. ${ }^{48,57,62,72}$ There are reports of the benefits of colchicine. ${ }^{73,74}$

As seen in bullous pemphigoid, there are some reported cases of therapeutic success with macrolides, such as erythromy- cin, and with the combination of tetracycline and nicotinamide. ${ }^{75,76}$ There are others antimicrobial agents that can be used, such as trimethoprim-sulfamethoxazole and oxacillin. ${ }^{48}$

Because these drugs fail and because some patients develop unwanted side effects, mainly related to the long-term use of corticosteroids and immunosuppressants, relatively new and safe therapeutic strategies, such as intravenous immunoglobulin and immunoadsorption, have been used successfully in recent years. ${ }^{48}$

There is a case report that described the use of rituximab. ${ }^{77}$

Systemic therapy is required until patients enter complete clinical remission, after which maintenance of the drug dosages should be adjusted according to the clinical evaluation of mucocutaneous lesions. In case of recurrence, systemic therapy should be restarted and continued over weeks or months after the complete disappearance of all lesions. ${ }^{48}$

\section{EVOLUTION AND PROGNOSIS}

The natural course of the disease is characterized by persistent lesions for several years, with remission occurring in many patients. ${ }^{63,64}$

Among children, remission usually takes place before puberty, although there are cases that persist into adulthood. ${ }^{64}$ When the disease endures in adolescence, it is usually milder than the initial presentation. ${ }^{48}$

In general, the blisters do not leave scars; alterations in pigmentation are more frequent, such as residual hyperchromia and hypochromia. ${ }^{63,64}$ There seems to be no correlation between the severity of the clinical presentation and disease chronicity. ${ }^{63}$

A small number of children and adults have exceptionally severe mucosal disease that progresses into cicatricial conjunctivitis and even blindness. ${ }^{72}$ At University Hospital of the Federal University of Santa Catarina, there was a case of a 47 -year-old female patient with LABD who began follow-up in the dermatology service in 2012, presenting with a clinical condition that began 19 years ago, with mucocutaneous involvement, primarily the ocular mucosa. On the hospital admission, she had amaurosis of the left eye and significant visual loss in the right eye, with a history of previous systemic treatments (dapsone, corticotherapy, sulfamethoxypyridazine, colchicine, mycophenolate mofetil, rituximab). It was started with monthly pulses of cyclophosphamide in combination with systemic corticosteroid therapy, with lesion control and follow-up with the ophthalmology team. Although the disease was controlled with cyclophosphamide, alternative systemic therapies were considered, due to the risks of the chronic use of cyclophosphamide, especially the onset of malignancies, such as bladder cancer.

\section{CONCLUSION}

Linear IgA dermatosis is a rare bullous disease that is often confused with other bullous diseases, such as bullous pemphigoid and dermatitis herpetiformis, due to the heterogeneity of its clinical presentation. In all patients who appear to present with LABD, a biopsy with DIF should be performed for diagnostic confirmation and initiation of the correct treatment.] 


\section{REFERENCES}

1. Collin P, Salmi TT, Hervonen K, Kaukinen K, Reunala T. Dermatitis herpetiformis: a cutaneous manifestation of coeliac disease. Ann Med. 2017;49:23-31.

2. Bolotin D, Petronic-Rosic V. Dermatitis herpetiformis. Part I. Epidemiology, pathogenesis, and clinical presentation. J Am Acad Dermatol. 2011;64:1017-24.

3. Cardones AR, Hall RP 3rd. Pathophysiology of dermatitis herpetiformis: a mode for cutaneous manifestations of gastrointestinal inflammation. Dermatol Clin. 2011;29:469-77.

4. Kárpáti S. Dermatitis herpetiformis. Clin Dermatol. 2012;30:56-9.

5. Tu H, Parmentier L, Stieger M, Spanou Z, Horn M, Beltraminelli H, e tal. Acral purpura as leading clinical manifestation of dermatitis herpetiformis: report of two adult cases with a review of the literature. Dermatology. 2013;227:1-4.

6. Jakes AD, Bradley S, Donlevy L. Dermatitis herpetiformis. BMJ. 2014 Apr 16;348:g2557

7. Kárpáti S. An exception within the group of autoimmune blistering diseases: dermatitis herpetiformis, the gluten-sensitive dermopathy. Dermatol Clin 2011:29:463-8.

8. Herrero-González JE. Clinical guidelines for the diagnosis and treatment of dermatitis herpetiformis. Actas Dermosifiliogr. 2010;101:820-6.

9. Lewis NR, Logan RF, Hubbard RB, West J. No increase in risk of fracture, malignancy or mortality in dermatitis herpetiformis: a cohort study. Aliment Pharmacol Ther. 2008:27:1140-7.

10. Fry L. Dermatitis herpetiformis: problems, progress and prospects. Eur Dermatol. 2002:12:523-31.

11. Nicolas ME, Krause PK, Gibson LE, Murray JA. Dermatitis herpetiformis. Int J Dermatol. 2003;42:588-600.

12. Warren SJ, Cockerell CJ. Characterization of a subgroup of patients with dermatitis herpetiformis with nonclassical histologic features. Am J Dermatopathol. 2002;24:305-8

13. Caproni M, Antiga E, Melani L, Fabbri $\mathrm{P}$; Italian Group for Cutaneous Immunopathology. Guidelines for the diagnosis and treatment of dermatites herpetiformis. J Eur Acad Dermatol Venereol. 2009;23:633-8.

14. Alonso-Llamazares J, Gibson LE, Rogers RS 3rd. Clinical, pathologic, and immunopathologic features of dermatitis herpetiformis: review of the Mayo Clinic experience. Int J Dermatol. 2007;46:910-9.

15. Antiga $\mathrm{E}$, Caproni $\mathrm{M}$. The diagnosis and treatment of dermatitis herpetiformis. Clin Cosmet Investig Dermatol. 2015:8:257-65.

16. Hill PG, Holmes GK. Coeliac disease: a biopsy is not always necessary for diagnosis. Aliment Pharmacol Ther. 2008;27:572-7.

17. Ingen-Housz-Oro S, Joly P, Bernard P, Bedane C, Prost C; Centres de référence des maladies bulleuses auto-immunes. Société Française de Dermatologie. Recommandations des centres de référence des maladies bulleuses autoimmunes pour le diagnostic et la prise en charge de la dermatite herpétiforme. Dermatitis herpetiformis. Guidelines for the diagnosis and treatment. Ann Dermato Venereol. 2011;138:271-3

18. Cardones AR, Hall RP 3rd. Management of dermatitis herpetiformis. Dermato Clin. 2011;29:631-5

19. Ciacci C, Ciclitira P, Hadjivassiliou M, Kaukinen K, Ludvigsson JF, McGough N, et al. The gluten-free diet and its current application in coeliac disease and dermatitis herpetiformis. United European Gastroenterol J. 2015;3:121-35.

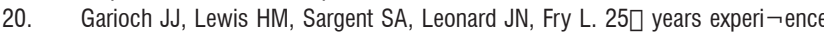
of a gluten-free diet in the treatment of dermatitis herpetiformis. Br J Dermatol. 1994:131:541-5

21. Ljunghall K, Tjernlund U. Dermatitis herpetiformis: effect of gluten-restricted and gluten-free diet on dapsone requirement and on IgA and C3 deposits in uninvolved skin. Acta Derm Venereol. 1983;63:129-36.

22. Fry L, Leonard JN, Swain F, Tucker WF, Haffenden G, Ring N, et al. Long term follow-up of dermatitis herpetiformis with and without dietary gluten withdrawal. Br J Dermatol. 1982;107:631-40.

23. Zhu YI, Stiller MJ. Dapsone and sulfones in dermatology: overview and update. J Am Acad Dermatol. 2001;45:420-34.

24. Coleman MD. Dapsone: modes of action, toxicity and possible strategies for increasing patient tolerance. Br J Dermatol. 1993;129:507-13

25. Prussick R, Ali MA, Rosenthal D, Guyatt $G$. The protective effect of vitamin $E$ on the hemolysis associated with dapsone treatment in patients with dermatitis herpetiformis. Arch Dermatol. 1992;128:210-3.

26. Barclay JA, Ziemba SE, Ibrahim RB. Dapsone-induced methemoglobinemia: a primer for clinicians. Ann Pharmacother. 2011:45:1103-15.

27. Rhodes LE, Tingle MD, Park BK, Chu P, Verbov JL, Friedmann PS. Cimetidine improves the therapeutic/toxic ratio of dapsone in patients on chronic dapsone therapy. Br J Dermatol. 1995;132:257-62.

28. Wozel G, Blasum C. Dapsone in dermatology and beyond. Arch Dermatol Res. 2014;306:103-24

29. Kahn G. Dapsone is safe during pregnancy. J Am Acad Dermatol. 1985;13:838-9
30. Bolotin D, Petronic-Rosic V. Dermatitis herpetiformis. Part II. Diagnosis, management, and prognosis. J Am Acad Dermatol. 2011;64:1027-33.

31. Goldstein BG, Smith JG Jr. Sulfasalazine in dermatitis herpetiformis. J Am Acad Dermatol. 1990;22:697.

32. Willsteed E, Lee M, Wong LC, Cooper A. Sulfasalazine and dermatitis herpetiformis. Australas J Dermatol. 2005;46:101-3.

33. Silvers DN, Juhlin EA, Berczeller PH, McSorley J. Treatment of dermatites herpetiformis with colchicine. Arch Dermatol. 1980;116:1373-84.

34. Stenveld HJ, Starink TM, van Joost T, Stoof TJ. Efficacy of cyclosporine in two patients with dermatitis herpetiformis resistant to conventional therapy. J Am Acad Dermatol. 1993;28:1014-5.

35. Shah SA, Ormerod AD. Dermatitis herpetiformis effectively treated with heparin, tetracycline and nicotinamide. Clin Exp Dermatol. 2000;25:204-5.

36. Zemtsov A, Neldner KH. Successful treatment of dermatitis herpetiformis with tetracycline and nicotinamide in a patient unable to tolerate dapsone. J Am Acad Dermatol. 1993;28:505-6

37. Kotze LM. Dermatitis herpetiformis, the celiac disease of the skin! Arg Gastroenterol. 2013;50:231-5

38. Albers LN, Zone JJ, Stoff BK, Feldman RJ. Rituximab treatment for recalcitrant dermatitis herpetiformis. JAMA Dermatol. 2017;153:315-18

39. Marakli SS, Uzun S, Ozbek S, Tuncer I. Dermatitis herpetiformis in a patient receiving infliximab for ankylosing spondylitis. Eur J Dermatol. 2008;18:88-9.

40. Bardella MT, Fredella C, Trovato C, Ermacora E, Cavalli R, Saladino V, et al. Longterm remission in patients with dermatitis herpetiformis on a normal diet. $\mathrm{Br} J$ Dermatol. 2003;149:968-71.

41. Paek SY, Steinberg SM, Katz SI. Remission in dermatitis herpetiformis: a cohort study. Arch Dermatol. 2011;147:301-5.

42. Grainge MJ, West J, Solaymani-Dodaran M, Card TR, Logan RF. The long-term risk of malignancy following a diagnosis of coeliac disease or dermatitis herpetiformis: a cohort study. Aliment Pharmacol Ther. 2012;35:730-9.

43. Lewis HM, Renaula TL, Garioch JJ, Leonard JN, Fry JS, Collin P, et al. Protective effect of gluten-free diet against development of lymphoma in dermatitis herpetiformis. Br J Dermatol. 1996;135:363-7.

44. Hervonen K, Alakoski A, Salmi TT, Helakorpi S, Kautiainen H, Kaukinen K, et al. Reduced mortality in dermatitis herpetiformis: A population-based study of 476 patients. Br J Dermatol. 2012;167:1331-7.

45. Reunala T, Salmi TT, Hervonen K, Kaukinen K, Collin P. Dermatitis Herpetiformis: A common extraintestinal manifestation of coeliac disease. Nutrients. 2018;10. pii: E602.

46. Guide SV, Marinkovich MP. Linear IgA Bullous Dermatosis. Clin Dermatol. 2001:19:719-27.

47. Egan CA, Zone JJ. Linear IgA bullous dermatosis. Int J Dermatol. 1999;38:818-27.

48. Fortuna G, Marinkovich MP. Linear immunoglobulin A bullous dermatosis. Clin Dermatol. 2012;30:38-50.

49. Kharfi M, Khaled A, Karaa A, Zaraa I, Fazaa B, Kamoun MR. Linear IgA bullous dermatosis: the more frequent bullous dermatosis of children. Dermatol Online J. 2010;16:2

50. Kridin K. Subepidermal autoimmune bullous diseases: overview, epidemiology, and associations. Immunol Res. 2018;66:6-17.

51. Zillikens D, Wever S, Roth A, Weidenthaler-Barth B, Hashimoto T, Bröcker EB. Incidence of autoimmune subepidermal blistering dermatoses in a region of central Germany. Arch Dermatol. 1995;131:957-8.

52. Wojnarowska F, Bhogal BS, Black MM. Chronic bullous disease of childhood and linear IgA disease of adults are IgA1-mediated diseases. $\mathrm{Br} J$ Dermatol. 1994:131:201-4

53. Zillikens D. BP 180 as the common autoantigen in blistering disease with different clinical phenotypes. Keio J Med. 2002:51:21-8

54. Braun-Falco 0, Plewig G, Wolff HH, Burgdorf WHC. Blistering diseases. In: BraunFalco 0, Plewig G, Wolff HH, Burgdorf WHC. Dermatology. 2 ed. Milano: SpringerVerlag; 2000. p. 648-95.

55. Kanagusuko T, Aoki V, Tebcherani AJ, Sanchez APG. Linear IgA dermatosis induced by pregnancy. An Bras Dermatol. 2008;83:49-52.

56. Mutasim DF, Adams BB. Immunofluorescence in dermatology. J Am Acad Dermatol. 2001;45:803-22

57. Zone JJ. Clinical Spectrum, Pathogenesis and Treatment of Linear IgA Bullous Dermatosis. J Dermatol. 2001;28:651-3.

58. Caproni M, Rolfo S, Bernacchi E, Bianchi B, Brazzini B, Fabbri P. The role of lymphocytes, granulocytes, mast cells and their related cytokines in lesional skin of linear IgA bullous dermatosis. Br J Dermatol. 1999;140:1072-8.

59. Chanal J, Ingen-Housz-Oro S, Ortonne N, Duong TA, Thomas M, Valeyrie-Allanore $\mathrm{L}$, et al. Linear IgA bullous dermatosis: comparison between the drug-induced and spontaneous forms. Br J Dermatol. 2013;169:1041-8. 
60. Salmhofer W, Soyer HP, Wolf P, Födinger D, Hödl S, Kerl H. UV light-induced linear IgA dermatoses. J Am Acad Dermatol. 2004;50:109-15.

61. Wojnarowska F, Venning VA. Immunobullous diseases. In: Burns T, Breathnach S, Cox N, Griffiths C, editors. Rook's Textbook of Dermatology. 8th ed. Oxford: Wiley-Blackwell; 2010. p. 40-51.

62. Machado TYS, Enokihara MMSS, lida TM, Porro AM, et al. Adult linear IgA bullous dermatosis: report of three cases. An Bras Dermatol. 2018:93:435-7.

63. Miyamoto D, Maragno L. Dermatose Bolhosa por IgA Linear. In: Belda Junior W, Di Chiacchio N, Criado PR, editores. Dermatoses Bolhosas Autoimunes. São Paulo: Atheneu; 2016. p. 85-92.

64. Venning VA. Linear IgA Disease: Clinical Presentation, Diagnosis, and Pathogenesis. Immunol Allergy Clin N Am. 2012;32:245-53.

65. Antiga E, Bellandi S, Bianchi B, Del Bianco E, Pierini I, Cozzani E, et al. A further case of subacute prurigo-like linear IgA bullous dermatoses: growing evidence of a new subset. Int J Dermatol. 2012;51:1500-1.

66. Talhari C, Althaus C, Megahed M. Ocular linear IgA disease resulting in blindness. Arch Dermatol. 2006;142:786-7.

67. Pereira AR, Moura LHB, Pinheiro JRS, Pasin VP, Enokihara MMSS, Porro AM. Vancomycin-associated linear IgA disease mimicking toxic epidermal necrolysis. An Bras Dermatol. 2016;91(Suppl 1):S35-8.

68. Arbache ST, Nogueira TG, Delgado L, Miyamoto D, Aoki V. Immunofluorescence testing in the diagnosis of autoimmune blistering diseases: overview of 10-year experience. An Bras Dermatol. 2014;89:885-9.
69. Aoki V, Sousa JX Jr, Fukumori LM, Périgo AM, Freitas EL, Oliveira ZN. Imunofluorescência direta e indireta. An Bras Dermatol. 2010;85:490-500.

70. Morrison LH. Direct immunofluorescence microscopy in the diagnosis of autoimune bullous dermatoses. Clin Dermatol. 2001;19:607-13.

71. Souza BC, Fregonesi NC, Tebcherani AJ, Sanchez AP, Aoki V, Fernandes JC. Dermatose por IgA linear - relato de um caso exuberante. An Bras Dermatol. 2013:88(Suppl 1):S67-70.

72. Ng SY, Venning VV. Management of Linear IgA Disease. Dermatol Clin 2011;29:629-30.

73. Benbenisty KM, Bowman PH, Davis LS. Localized linear IgA disease responding to colchicine. Int J Dermatol. 2002;41:56-8.

74. Ang P, Tay YK. Treatment of linear IgA bullous dermatosis of childhood with colchicine. Pediatr Dermatol. 1999:16:50-2.

75. Cooper SM, Powell J, Wojnarowska F. Linear IgA disease: successful treatment with erythromycin. Clin Exp Dermatol. 2002;27:677-9.

76. Peoples D, Fivenson DP. Linear IgA bullous dermatosis: successful treatment with tetracycline and nicotinamide. J Am Acad Dermatol. 1992;26:498-9.

77. Lozinski A, Baum S, Sagi L, Volkov A, Trau H, Barzilai A. Rituximab (Mabthera) for treatment of rare autoimmune bullous skin disorders. Harefuah. 2012;151:562-5.

\section{AUTHORS'CONTRIBUTIONS}

Everton Carlos Siviero Do Vale (iD) ORCID 0000-0002-9172-3639

Approval of the final version of the manuscript; conception and planning of the study; elaboration and writing of the manuscript; critical review of the literature; critical review of the manuscript

Oscar Cardoso Dimatos ～～０000-0002-2201-0387

Approval of the final version of the manuscript; elaboration and writing of the manuscript; critical review of the literature; critical review of the manuscript

Adriana Maria Porro $\quad$ (iD) ORCID 0000-0003-0736-4790

Approval of the final version of the manuscript; conception and planning of the study; elaboration and writing of the manuscript; Critical review of the literature; Critical review of the manuscript

Claudia Giuli Santi $\quad$ (iD) ORCID 0000-0003-3650-4254

Approval of the final version of the manuscript; conception and planning of the study; elaboration and writing of the manuscript; Critical review of the literature; Critical review of the manuscript

How to cite this article: Vale ECS, Dimatos OC, Porro AM, Santi CG. Consensus on the treatment of autoimmune bullous dermatoses: dermatitis herpetiformis and linear IgA bullous dermatosis - Brazilian Society of Dermatology. An Bras Dermatol. 2019;94(2 Suppl 1):S48-55. 